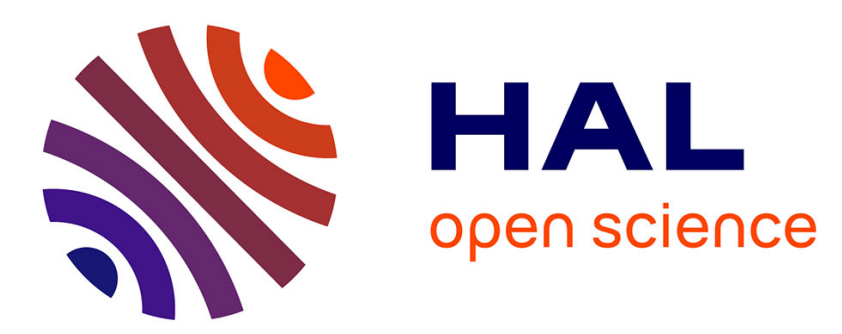

\title{
Exhaustive exploration of the conformational landscape of small cyclic peptides using a robotics approach
}

\author{
Maud Jusot, Dirk Stratmann, Marc Vaisset, Jacques Chomilier, Juan Cortés
}

\section{To cite this version:}

Maud Jusot, Dirk Stratmann, Marc Vaisset, Jacques Chomilier, Juan Cortés. Exhaustive exploration of the conformational landscape of small cyclic peptides using a robotics approach. Journal of Chemical Information and Modeling, 2018, 58 (11), pp.2355-2368. 10.1021/acs.jcim.8b00375 . hal-01893751

\section{HAL Id: hal-01893751 \\ https://hal.laas.fr/hal-01893751}

Submitted on 11 Oct 2018

HAL is a multi-disciplinary open access archive for the deposit and dissemination of scientific research documents, whether they are published or not. The documents may come from teaching and research institutions in France or abroad, or from public or private research centers.
L'archive ouverte pluridisciplinaire HAL, est destinée au dépôt et à la diffusion de documents scientifiques de niveau recherche, publiés ou non, émanant des établissements d'enseignement et de recherche français ou étrangers, des laboratoires publics ou privés. 


\title{
Exhaustive Exploration of the Conformational Landscape of Small Cyclic Peptides Using a Robotics Approach
}

\author{
Maud Jusot, ${ }^{\dagger, \ddagger}$ Dirk Stratmann, ${ }^{\dagger}$ Marc Vaisset,,$^{\ddagger}$ \\ Jacques Chomilier, ${ }^{*, \dagger}$ and Juan Cortés ${ }^{*, \ddagger}$ \\ †Sorbonne Université, MNHN, CNRS, IRD, Institut de Minéralogie, de Physique des \\ Matériaux et de Cosmochimie, IMPMC, 75005 Paris, France \\ $\ddagger L A A S-C N R S$, Université de Toulouse, CNRS, 31400 Toulouse, France \\ E-mail: jacques.chomilier@upmc.fr; juan.cortes@laas.fr
}

\begin{abstract}
Small cyclic peptides represent a promising class of therapeutic molecules with unique chemical properties. However, the poor knowledge of their structural characteristics makes their computational design and structure prediction a real challenge. In order to better describe their conformational space, we developed a method, named EGSCyP, for the exhaustive exploration of the energy landscape of small head-totail cyclic peptides. The method can be summarized by (i) a global exploration of the conformational space based on a mechanistic representation of the peptide and the use of robotics-based algorithms to deal with the closure constraint, (ii) an allatom refinement of the obtained conformations. EGSCyP can handle D-form residues and N-methylations. Two strategies for the side-chains placement were implemented and compared. To validate our approach, we applied it to a set of three variants of cyclic RGDFV pentapeptides, including the drug candidate Cilengitide. A comparative
\end{abstract}


analysis was made with respect to replica exchange molecular dynamics simulations in implicit solvent. It results that the EGSCyP method provides a very complete characterization of the conformational space of small cyclic pentapeptides.

\section{Introduction}

After a relative decline for decades, peptides are turning back to the light as promising therapeutics drugs. ${ }^{1}$ This is partially due to advances in chemistry to produce stable cyclic peptides as well as to the development of biotechnologies avoiding most of the drawbacks objected to peptides such as enzyme digestion or membrane penetration. ${ }^{2-6}$ Peptides combine the advantages of proteins and small molecules: they have high selectivity as proteins, and metabolic stability, oral availability and low immunogenicity, as small molecules. ${ }^{2,6-8}$ Compared to small ligands, peptides can occupy larger surfaces of interaction and reach higher specificities. ${ }^{2,6,7,9,10}$ Thus, a particularly interesting application of peptides is the inhibition of protein-protein interactions involved in severe human diseases, including cancers and neurodegenerative disorders. ${ }^{11-13}$ Therapeutic peptides are (re-)designed, aiming at improving their stability as well as their resistance to degradation by proteases. This can be achieved by cyclization and other chemical modifications, such as N-methylations or the use of D-amino acids. ${ }^{3,14-18}$ Interestingly, combining cyclization and N-methylation has proven to improve membrane permeability, which is critical in the development of therapeutics against intracellular targets. ${ }^{7}$ It has recently been shown that cyclisation can result in a four orders of magnitude increase in the binding affinity for their target. ${ }^{19}$ Finally, it has been shown that the N-methylation can modulate both the affinity and the specificity of a peptide for its target. ${ }^{20}$ Therefore, cyclic peptides and their chemical modifications present pharmaceutical advantages that support the importance of their in silico design.

In spite of these promising properties in the field of pharmacology, we still are at the dawn of a sound understanding of cyclic peptides, in particular for head-to-tail cyclization, which would open the road to in silico predictions of their 3D structures. Indeed, there 
are only a few tens of structures annotated as "cyclic peptide" in the Protein Data Bank (PDB) and in the Cambridge Structural Database, as far as the length is less than 50 amino acids. Thus, template-based homology modeling methods are not adequate and only ab initio modeling is feasible. Besides, most of the de novo methods for modeling proteins are not adapted to cyclic peptides. Recent efforts have been made to develop or to adapt structure prediction tools to cyclic peptides. However, few of them are able to treat small headto-tail cyclic peptides (with less than seven residues) involving chemical modifications. For example, PEP-FOLD ${ }^{21,22}$ does not deal with head-to-tail cyclic peptides. PEPstrMOD ${ }^{23}$ and I-TASSER ${ }^{24}$ do not deal with sequences shorter than seven residues. The method "Simple Cyclic Peptide Prediction" of $\operatorname{Rosetta}^{8}$ can treat small peptides but cannot deal with Nmethylation, so far. To our knowledge, only Peplook ${ }^{25}$ can deal with mixed-chiral small cyclic peptides and N-methylation. The difficulty to develop tools for predicting the structure of small cyclic peptides is essentially due to their very constrained structure that cannot contain any secondary structure nor hydrophobic core, ${ }^{15,26,27}$ therefore with $\phi$ and $\psi$ dihedral angles tending to fall outside the canonical allowed regions observed in the Ramachandran diagram ${ }^{28}$ for proteins. ${ }^{29}$ In addition, the use of D-amino acids and N-methylations makes the modeling even more difficult. ${ }^{30}$ To improve structure prediction, there is a real necessity of better understanding the conformational landscape of small cyclic peptides, and the related dihedral range needed to describe it. Our ambition was thus to explore the energy landscape of short, modified head-to-tail cyclic peptides. Indeed, while structure prediction aims at finding the most stable or probable conformations, global exploration methods are aimed to provide an overall picture of the conformational space.

Although molecular dynamics simulations and Monte Carlo methods can be used to explore the conformational space of linear peptides, ${ }^{31,32}$ the application of these methods to cyclic peptides is less straightforward. This is mainly due to the ring-closure constraint, which leads to high energy barriers between the different meta-stable conformations. ${ }^{11,33,34}$ Thus, it makes the conformational sampling very challenging. Actually, even for small sys- 
tems, achieving a complete exploration of the energy landscape requires long simulation times when using basic approaches. Advanced methods are required to overcome this difficulty. For example, Replica Exchange Molecular Dynamics (REMD) simulations have been applied to cyclic peptides. ${ }^{35}$ Metadynamics is a valuable alternative, ${ }^{11}$ but the parametrization of the simulation, i.e., the selection of the collective variables that are critical to capture the degrees of freedom (DoF) of the system, is still a bottleneck. Very recently, the accelerated molecular dynamics methodology has been successfully applied to cyclic peptides. ${ }^{19}$ However, these simulations depend on system-specific boosting parameters, and are very computationally expensive. Therefore, there is a need for alternative approaches, adapted to cyclic peptides, for an unambiguous complete exploration of the conformational landscape, getting rid of the difficult assessment of convergence necessary in the various methods based on molecular dynamics.

Numerous methods have been proposed since the the seminal work of Go and Scheraga ${ }^{36}$ to efficiently sample cyclic molecules. We can mention for instance the work of Wu and Deem, ${ }^{37}$ and of Coutsias et al. ${ }^{38}$ Inspired from these methods, we present a robotics-based approach for exploring the conformational landscape of head-to-tail cyclic peptides possibly involving mixed-chirality and N-methylated residues. Our method, called Exhaustive Grid Search for Cyclic Peptides (EGSCyP), is based on a multi-level representation of the peptide, and on the application of different algorithms at the various levels. Backbone conformations are first exhaustively sampled considering dihedral angles as the main variables. An inverse kinematics (IK) algorithm ${ }^{39}$ is used at this level to enforce loop closure. Then, for each backbone conformation, side-chains are placed and local minimization is performed using an all-atom representation. In this paper, we focused on pentapeptides, but our method also applies to tetrapeptides and can be trivially adapted to hexapeptides provided a simple parallelization of the algorithm.

In order to validate this approach, we sampled the conformational landscape of a set of three cyclic pentapeptides described in the literature, ${ }^{40,41}$ containing the widely studied RGD 
motif. One of these peptides is Cilengitide, ${ }^{42}$ which is an example of promising N-methylated RGD cyclic pentapeptide, developed by the Kessler group as inhibitor of protein-protein interaction of the $\alpha \mathrm{V} \beta 3$ and the $\alpha \mathrm{V} \beta 5$ integrins. It has reached the phase III of clinical trial for glioblastomas and is also under evaluation for other types of cancer. ${ }^{43}$ This pentapeptide was chosen as a proof of concept since one structure is deposited and it is well studied experimentally. To evaluate the completeness and the accuracy of the EGSCyP approach, the results on these three cyclic peptides were compared with those obtained with REMD simulations.

\section{Methods}

\section{Overview}

EGSCyP applies a kind of "divide and conquer" paradigm. The global conformational exploration problem is divided into several sub-problems, each of which involves different variables. The backbone dihedral angles are treated first, since they are the most important degrees of freedom of a peptide. Among the backbone dihedral angles, the $\omega$ angles (corresponding to peptide bonds) are particularly rigid. Therefore, and aiming to reduce the combinatorial complexity, their values are randomly sampled from a Gaussian distribution centered at $180^{\circ}$, rather than systematically explored. The exhaustive exploration focuses on the $\phi$ and $\psi$ angles. The loop-closure constraint imposes a non-linear relationship between these angles. More precisely, the value of 6 angles is determined from the value of the other $n-6$ angles $(n$ representing the total number of $\phi$ and $\psi$ dihedral angles), using an inverse kinematics (IK) solver. In our approach, we assign these 6 dependent variables to the $\phi$ and $\psi$ angles of three consecutive residues. Therefore, for a pentapeptide backbone, the remaining (independent) variables to be sampled are the $\phi$ and $\psi$ of only two residues. Thanks to the low dimension, these four variables can be sampled with high resolution using an exhaustive grid search. The conformation of the side-chains is then sampled for each backbone conformation satis- 
fying loop closure and without significant steric clashes. We have investigated two different approaches for solving this second sub-problem. Finally, the whole conformation is locally minimized at an all-atom level (i.e. considering all the degrees of freedom simultaneously). The multi-level model and the different stages of the EGSCyP algorithm are explained with more detail below.

\section{Molecular model}

The representation of molecules is generally based either on the Cartesian coordinates of all their atoms, or on the set of internal coordinates corresponding to the relative positions of their covalently bonded atoms. This second representation can be defined by three types of DoF: bond lengths, bond angles and dihedral angles. It has been shown that the first two present low variations at room temperature. ${ }^{44}$ Therefore, the model can be simplified considering these parameters as constants, adopting the rigid geometry assumption, ${ }^{45}$ which means that the only DoF are the dihedral angles. This representation of a peptide allows modeling it as an articulated mechanism, similar to a robotic $\operatorname{arm}^{39}$ (see Figure 1). Thanks to this choice of representation, algorithms from robotics can be applied to explore the conformational space of molecules. ${ }^{46-48}$ The approach we present in this paper uses both models: (1) the mechanistic one for the global exploration of the backbone conformation and the side-chain placement, (2) the Cartesian all-atom model for the refinement with the relaxation method.

\section{Sampling algorithm}

The EGSCyP algorithm is summarized in Figure 2 for a pentapeptide. The exploration starts by the selection of two consecutive residues to be sampled, involving two pairs of $\phi$ $\psi$ angles.

By default, the selected residues are the first and the last ones in the PDB file given as input (see dataset paragraph). In the following, these two residues are named $I$ and $V$, 
(a)

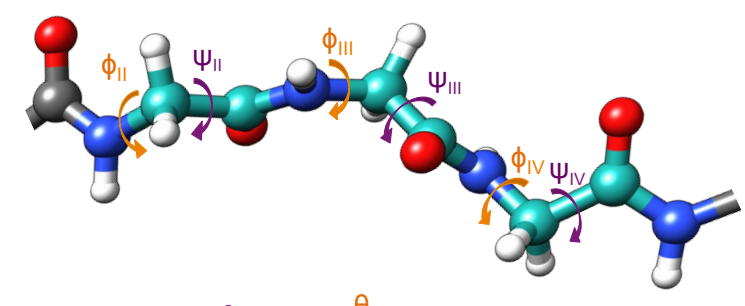

(b)

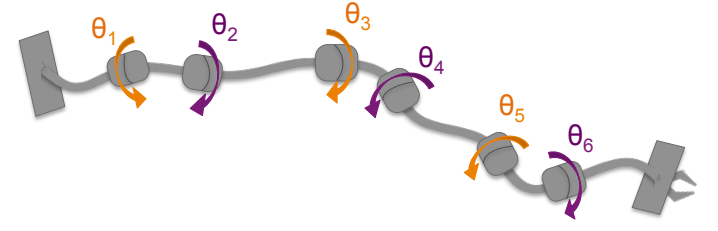

Figure 1: Analogy between a tripeptide (a) and a robotic arm (b). The dihedral angles $\phi$ (orange arrows) and $\psi$ (purple arrows) of the tripeptide (in cyan) correspond to the revolute joints of the kinematic chain in this analogy.

respectively. The effect of the choice of the two sampled residues has been evaluated by testing all the possible positions (see results section). The sampling of all possible combinations of the two pairs of $\phi-\psi$ dihedral angles is made with a constant step size of $10^{\circ}$ over the range from $-180^{\circ}$ to $180^{\circ}$. Then, for every combination, the five $\omega$ angles are randomly selected from a Gaussian distribution with a standard deviation of $10^{\circ}$ centered around $180^{\circ}$. Then, the IK method is applied to close the cycle between the sampled residues (see below for more details). In other words, the IK method finds feasible values for the $\phi-\psi$ angles of residues $I I, I I I$ and $I V$. If there is no solution, the $\omega$ angles are sampled again until a solution is found or until a maximum number of iterations is reached (100 iterations in our implementation). If no solution is finally found, the next combination of torsion angles is tested. Otherwise, for each solution of the IK method, possible collisions between the backbone atoms (and the methyl carbon of N-methylated residues, if there was one) are checked. A collision is detected if the distance between two non-bonded atoms is less than $60 \%$ of the sum of their van der Waals radii ${ }^{49}$ thus accepting a small overlap. This geometric filtering 

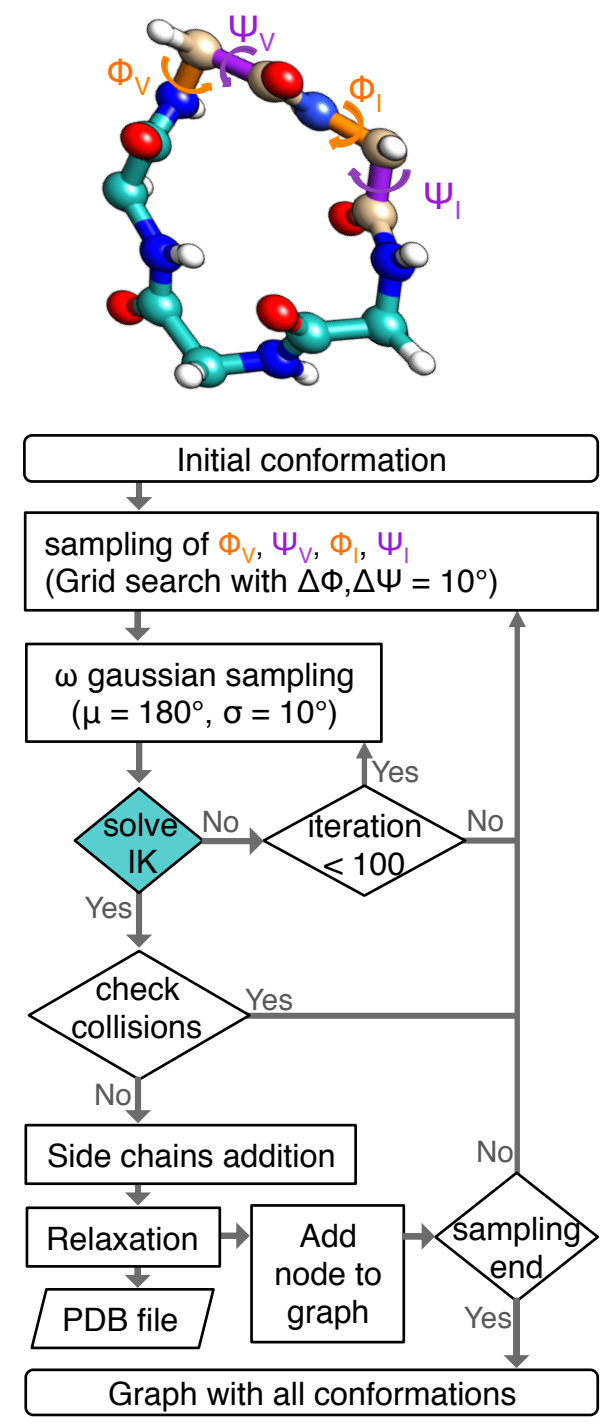

Figure 2: Flowchart of the EGSCyP algorithm. The backbone of a pentapeptide is represented to illustrate the sampled dihedral angles $\phi$ and $\psi$ colored in orange and purple respectively. The tripeptide on which the IK is applied is colored in cyan. 
was not chosen too restrictive, because the final relaxation could avoid small collisions not filtered at this step. Then, in absence of collision, the side chains are positioned using one of the following methods: (1) the SCWRL4 software, ${ }^{50}$ or (2) a variant of the Basin Hopping (BH) method. ${ }^{51}$ Details about these methods are described below in the paragraph about side chains placement. Finally, a few steps of structural relaxation (detailed below) are performed using Sander from AmberTools 16. ${ }^{52}$ This last stage allows releasing constraints imposed by the rigid geometry representation. The relaxation is made with the amberff96 force field ${ }^{53}$ with a Generalized Born implicit solvent (all default parameters were set on). The choice of this force field and implicit solvent was made based on the reasonable performance of this combination on peptides compared to other force fields. ${ }^{54}$ During relaxation, the $\phi$ and $\psi$ dihedral angles from the two sampled residues are restrained to their sampled values. For the calculations presented below, the maximum number of cycles of minimization was set to 1000, with 500 steps of steepest descent followed by conjugate gradient. The convergence energy criterion for the minimization was set to $0.1 \mathrm{kcal} / \mathrm{mol}-\AA$. At the end of each iteration, for each solution, a conformation is built (i.e., atomic coordinates are extracted) and the dihedral angles values are recorded for all the residues as well as the energy of the peptide, calculated by Amber. A connectivity graph is created during the sampling process, with one node for each conformation. In the sampling grid, neighbor nodes are linked in such a way that each node is connected to all the nodes having the four sampled dihedral angles within a window of $\pm 10^{\circ}$. As explained below, the connectivity graph is used within the side-chain placement method.

\section{Inverse Kinematics}

As previously described, a molecule can be modeled as an articulated mechanism. Using standard conventions applied in robotics, such as the modified Denavit-Hartenberg convention ${ }^{55}$ used in this work, a Cartesian coordinate system $F_{i}$ is attached to each rigid body, which corresponds to a small group of bonded atoms. Then, the relative location 
of two consecutive frames in the chain can be defined by a homogeneous transformation matrix ${ }^{i-1} T_{i}$. Assuming constant bond lengths and bond angles, the only variable parameter in this matrix corresponds to a bond torsions, $\theta_{i}$. In the case of a peptide backbone, and if we assume that the $\omega$ angles are also fixed to a given (sampled) value, the variables are the $\phi$ and $\psi$ angles. Thus, as illustrated in Figure 1, the backbone conformation for a tripeptide composed of residues $I I, I I I, I V$ is defined by the vector of six angles $\left\{\theta_{1}, \theta_{2}, \theta_{3}, \theta_{4}, \theta_{5}, \theta_{6}\right\}=\left\{\phi_{I I}, \psi_{I I}, \phi_{I I I}, \psi_{I I I}, \phi_{I V}, \psi_{I V}\right\}$.

The IK problem relies in finding values for this vector of angles such that they solve the following matrix equation: ${ }^{0} T_{1}{ }^{1} T_{2}{ }^{2} T_{3}{ }^{3} T_{4}{ }^{4} T_{5}{ }^{5} T_{6}={ }^{0} T_{6}$, where ${ }^{0} T_{6}$ is the homogeneous transformation matrix representing the relative pose of the first frame $F_{0}$ and the last frame $F_{6}$. In our case, $F_{0}$ and $F_{6}$ are determined from the conformation of the other two residues in the cyclic peptide, which were exhaustively sampled as explained in the previous section.

For solving the IK problem, we apply the method proposed by Renaud. ${ }^{56,57}$ This semianalytical solver, based on algebraic elimination theory, is rooted in the work of Lee and Liang. ${ }^{58,59}$ Our implementation incorporates ideas proposed by Manocha and Canny ${ }^{60}$ to improve numerical robustness. The solver is very computationally efficient, requiring about 0.1 milliseconds on a single processor. It was successfully applied in previous works on protein and polymer modeling. ${ }^{61-63}$ Note however that our approach does not rely on a particular IK solver. Other methods available in the literature could be applied (e.g. ${ }^{38,60,64}$ ). Nevertheless, we recommend the application of (semi-)analytical methods, which, in the present application case, have several important advantages with respect to purely numerical approaches such as Cyclic Coordinate Descent (CCD). ${ }^{65}$ The main advantage is that they simultaneously provide all the solutions to the IK problem: up to 16 for an articulated mechanism with six revolute joints, as our tripeptide model. In addition, they provide the exact solution in a single iteration, not suffering from slow convergence issues. 


\section{Side-chain placement}

Two methods were implemented and evaluated for the side-chain positioning. The first one was SCWRL4, ${ }^{50}$ a widely-used and efficient tool for the prediction of side-chain conformations, based on a rotamer library. In our case, the applicability of this method is limited, because it cannot deal with the N-methylated residues (it replaces them by glycines). Damino acids are not represented in the rotamer library either. Moreover, SCWRL4 does not deal with cyclization: collisions usually happen between the side-chains of the first and the last residues. Within our multi-stage exploration approach, the subsequent all-atom relaxation (performed with Amber) can solve some of these problems. However, wrong side-chain conformations may remain. Finally, despite the fairly high speed of SCWRL4, its use as a third-party program requiring system calls slows down the overall computing time.

The second approach used for the side-chain placement is much more general. It is based on stochastic optimization methods. More precisely, we applied a variant of the MonteCarlo-minimization method, ${ }^{66}$ also known as Basin Hopping (BH), ${ }^{51}$ as explained in previous work. ${ }^{67}$ This global optimization method consists of iteratively applying a random structural perturbation (global search) followed by a local energy minimization. At each iteration, the new local minimum is compared with the one generated in the previous iteration, and it is accepted or rejected based on the classical Metropolis criterion. In our implementation, the structural perturbation was applied to a randomly selected number of $\chi$ angles in a first step. Then, the local minimization based on a simple Monte-Carlo (MC) method at very low temperature (in order to remain in the local basin), applied small random perturbations to all the $\chi$ angles. Although this type of local minimization is in general less computationally efficient than gradient-based approaches usually applied within $\mathrm{BH}$, it is also less sensitive to small local traps. It requires about 10 seconds on a single processor (it is the most expansive step of our algorithm).

In order to decrease the overall computational cost, the BH method was not systematically applied to place the side-chains for every sampled conformation of the peptide back- 
bone. When a neighboring conformation of the peptide (i.e., a neighbor in the connectivity graph) has already been generated by the exhaustive exploration algorithm, the side-chains conformation was then generated by local minimization from this neighbor, using the local Monte-Carlo search. The computational time is reduced to 10 milliseconds and is equivalent to the use of SCWRL4. Energy evaluation within the side-chain placement procedure was performed using the same force field as in the subsequent relaxation process: the amberff96 force field with a Generalized Born implicit solvent.

\section{REMD}

For comparison, Replica Exchange Molecular Dynamics simulations were performed with GROMACS 5.1.2. ${ }^{68}$ As in the EGSCyP method, the simulations were performed using the OBC (Onufriev, Bashford, and Case) ${ }^{69}$ GBSA implicit solvent model and the Amber96 force field. ${ }^{53}$ A short minimization was first done with an alternation of one step of steepest descent step every 500 conjugate gradient steps. The maximum number of steps was set to 50,000 with a step size of $0.01 \mathrm{~nm}$ and an energy convergence criterion of $10 \mathrm{~kJ} / \mathrm{mol} . \mathrm{nm}$. The thermalizations and simulations were made with eight replica varying in temperature from $300 \mathrm{~K}$ to $450 \mathrm{~K}$ (at $300 \mathrm{~K}, 313 \mathrm{~K}, 329 \mathrm{~K}, 347 \mathrm{~K}, 367 \mathrm{~K}, 391 \mathrm{~K}, 418 \mathrm{~K}, 450 \mathrm{~K}$ ). The temperatures of the replica were chosen to keep constant the probability of accepting exchanges. Only a short phase of thermalization was realized on 100 ps (50 000 steps). Then, the REMD simulations were performed with parameters inspired from the work of Wakefield et al. ${ }^{35}$ The exchanges between neighboring replicas were made every 10 ps. Each replica ran for $2.4 \mu \mathrm{s}$, yielding a total simulation time of $19.2 \mu \mathrm{s}$ per peptide. The atomic coordinates were recorded every 10 ps. However, contrary to the work of Wakefield et al., ${ }^{35}$ we did not change the torsion scaling parameters to lower the $\omega$ angle torsional barriers and to accelerate the cis/trans sampling of $\mathrm{N}$-methylated residues. We kept it to the default value, because the cis conformations were only populated at a few percent in the study of Wakefield et al. ${ }^{35}$ 


\section{Clustering}

In order to rapidly and easily identify the main energy minima produced by EGSCyP and REMD simulations, we developed a simple clustering method inspired from the one recently proposed by Hosseinzadeh et al. ${ }^{26}$ It is based on the energy of conformations and the Root Mean Square Deviation (RMSD) calculated upon the $\phi-\psi$ dihedral angles. ${ }^{70}$ For EGSCyP, the energy taken into account is the final energy computed by Amber at the end of the relaxation. For REMD simulations, the potential energy for each frame of the lower-temperature replica is computed with Gromacs. The global energy minimum is selected as the center of the first cluster. Then, the RMSD between this minimum and all the other conformations is computed. All the conformations presenting a RMSD inferior to a threshold are put into the first cluster. The choice of this threshold will be discussed below, together with the results. Next, the same procedure is applied again: among the remaining conformations, the structure with the lowest energy is selected as the center of the second cluster and the RMSD is again computed for creating the second cluster. The procedure is repeated until all the conformations were included into a cluster. This approach, unlike classical clustering based only on distances, may be biased toward low-energy basins. However, its application in the context of this study to find and compare the energy minima basins seems quite appropriate. As a final stage, the representative structures of clusters from both methods are minimized without any constraint with Amber in order to be comparable.

\section{Dataset}

In this study, we considered three RGD cyclic pentapeptides selected from the work of the Kessler group. ${ }^{40,41}$ The sequences of these peptides are presented in Table 1.

For both the REMD simulations and EGSCyP method, all the cyclic structures were generated using UCSF-Chimera. ${ }^{71}$ This tool allows modeling non-natural amino acids, such as D-amino acids or N-methylated ones. The cyclization was made head-to-tail, i.e., a peptidic bond was created between the N-terminus and the C-terminus amino acids. The 
Table 1: Dataset of cyclic pentapeptides. Lower case letters indicate D-amino acids and single quote are for the N-methylated ones. RGDfV' corresponds to Cilengitide (PDB ID: $1 \mathrm{~L} 5 \mathrm{G})$.

\begin{tabular}{c|c} 
Peptide sequence & Type \\
\hline RGDFV & natural \\
RGDfV & D residue \\
RGDfV' & D residue + N-methylated residue
\end{tabular}

exhaustive exploration process performed by EGSCyP is independent of the initial conformation. The topology files were created with Tleap from AmberTools 16, ${ }^{52}$ using the Amber ff96 force field. ${ }^{53}$ The Amber topologies and partial charges of the N-methylated residues were computed with the RED-Server. ${ }^{72}$ For the REMD simulations, the Amber topology files were converted into Gromacs topology files with Acpype ${ }^{73}$ and manually modified for compatibility with recent versions of Gromacs.

\section{Results and discussion}

\section{Exhaustive grid search}

We used the EGSCyP method for the three peptides presented in Table 1. The level of exploration of the conformational landscape resulting from the EGSCyP approach was compared to the one produced by REMD simulations, over $2.4 \mu$ s for each replica of each peptide. Due to the low number of DoF of a cyclic pentapeptide and the relatively long simulation time, the REMD sampling is sufficiently exhaustive to be compared with the one obtained with EGSCyP (the convergence of the simulations and the percentage of accepting exchange between replica were verified; see supporting information Figures S1, S2 and S3).

Choice of the sampled residues: We did a systematic test to verify that the choice of the two exhaustively sampled residues does not significantly affect the results of the EGSCyP method. It consisted of repeatedly applying the EGSCyP method, selecting each time a different combination of two consecutive residues to be exhaustively sampled. The test was 
applied to the RGDfV peptide (see Table 1 for nomenclature). The obtained landscapes are fairly similar, independently from the residues chosen to be sampled (detailed results are available in supporting information in Figure S4 and Table S4). The differences can be explained by four reasons: (1) the more exhaustive sampling performed for two pairs of $\phi$ and $\psi$ angles (with respect to the angles solved by IK), which enforces the exploration of high-energy areas in the corresponding projections; (2) the randomized sampling process of the $\omega$ and $\chi$ angles; (3) the resolution of the discretization (i.e., $10^{\circ}$ step size in the grid search) for the exploration of the two pairs of $\phi$ and $\psi$ angles; (4) the imposed restrain on these four angles during the relaxation.

The first of these reasons explains why the percentage of coverage of the Ramachandran diagrams (defined as the percentage of $\phi, \psi$ pairs, within the grid spacing of $10^{\circ}$, for which at least one conformation was found by EGSCyP) varies from $85 \%$ to $95 \%$ for the residues solved by IK, while it grows from $97 \%$ to $98 \%$ for the two exhaustively sampled residues. In spite of these variations, the low energy basins still remain in the same areas of the Ramachandran plot. The main differences are localized in areas of high energy. Therefore, we can make the simplification of sampling any two successive residues and still obtain a very complete landscape.

Table 2 shows a summary of the results obtained with EGSCyP that are detailed on the next paragraphs.

IK Solutions: The sampling of the four $\phi$ and $\psi$ dihedral angles was realized with a step size of $10^{\circ}$, which means that $(360 / 10)^{4}=1.68 \times 10^{6}$ combinations were tested for each peptide. For each $\phi, \psi$ combination, up to 100 combinations of the five $\omega$ dihedral angles were tested. $20 \%-22 \%$ of the $1.68 \times 10^{6} \phi, \psi$ combinations (i.e, about 350,000 combinations) yielded at least one solution to the IK problem. This proportion is rather constant among the different peptides, which is due to the fact that, at this step, the side chains are not taken into account and the number of accepted conformations is thus independent from the sequence. Indeed, the results among these various sequences differ only by (1) the geometry 
Table 2: Summary of the performances of the EGSCyP method on the three cyclic pentapeptides. The RGDFV peptide is represented twice because of the two different methods used for side chain placement: SCWRL4 and the alternation of Basin Hopping (BH) and local minimization by Monte Carlo search (MC). The number of solutions of the IK problem is represented as a percentage of the cases with at least one solution among all the combinations of $\phi / \psi$ angles. The number of iterations for the $\omega$ angles sampling correspond to the number of iterations before the IK solver found a solution (until 100 iterations, in which case the $\phi / \psi$ combination is rejected). The percentage of collisions corresponds to the percentage of conformations among all the IK solutions containing overlapping atoms.

\begin{tabular}{l|c|c|c|c} 
Peptide sequence & RGDFV & RGDFV & RGDfV & RGDfV' \\
\hline Solutions found to IK problem & $21 \%$ & $21 \%$ & $22 \%$ & $20 \%$ \\
Number of iterations for $\omega$ sampling : & & & & \\
$\quad$ - Median & 6 & 6 & 5 & 3 \\
$\quad$ - First and third quartiles & $1-24$ & $1-24$ & $1-22$ & $0-11$ \\
Collisions found & $22 \%$ & $22 \%$ & $21 \%$ & $43 \%$ \\
Number of final conformations & 764,740 & 762,944 & 830,657 & 540,938 \\
Side Chain Methods & BH/MC & SCWRL4 & BH/MC & BH/MC \\
Side-chain conformations sampled by BH & $0.06 \%$ & - & $0.06 \%$ & $0.09 \%$
\end{tabular}

of the initial PDB structure, with the bond length and angles that can slightly vary between the initial structures (remember that they are kept fixed during the $\phi-\psi-\omega$ sampling and the use of IK), and (2) a random factor which can be fixed by the use of a unique random-seed for the sampling of the $\omega$ dihedral angles. The fact that less than $1 / 4$ of the $\phi$, $\psi$ combinations yielded IK solutions emphasizes the difficulty of ring closure for small cyclic peptides.

$\omega$ sampling: The median and the third quartile of the number of iterations over the combinations of the five $\omega$ angles sampling processed before a submission to the IK solver vary from 3 to 6 and from 11 to 24 respectively. Because solutions were generally found rapidly after a few iterations, the limit of 100 iterations is sufficient to find a solution, if any.

Number of collisions: The number of collisions between backbone atoms is rather constant (around $20 \%$ of the solutions found by IK per peptide) except for Cilengitide (RGDfV'). For this last peptide, the number of collisions increased up to $43 \%$. This is the consequence of the N-methylated valine residue which very easily enters in collision with the backbone due to the small size of the cyclic pentapeptide. This illustrates the fact that N-methylations 
constraints the structure of peptides and reduces their conformational landscape.

Side-Chain placement: Among the three considered pentapeptides, RGDFV is the only one involving no chemical modification. Therefore, we used this molecule to compare the performance of SCWRL4 and the BH method (see below). The combination of BH and local minimization from a neighbor conformation was applied for the two other peptides of Table 1 with side chains. Note that the percentage of conformations for which $\mathrm{BH}$ was applied was very low. For a large majority of the conformations (around 99\%), the side chains were placed by local minimization from a neighbor conformation in the connectivity graph. This shows that the graph is very dense, thanks to the exhaustiveness of the exploration.

Number of final conformations: The total number of conformations generated for each peptide is around 800,000, at the exception of Cilengitide (RGDfV') for which only 540,938 conformations were found (because of the collisions mentioned above). Note that the number of conformations is significantly larger than the number of sampled combinations of $\phi-\psi$ angles (around 350,000). The reason is that, in most of the cases, the IK solver finds several conformations for the tripeptide. Indeed, two IK solutions were found for around $50 \%$ of the combinations for which the closure constraint can be satisfied. Excepting one case, the IK solver found at most up to eight conformations for the tripeptide. The exact numbers of solutions found for each peptide are presented in supporting information (Table S3).

Time performance: An analysis of EGSCyP run time performance was made on Cilengitide. The total run time as well as the time for each stage of the algorithm were evaluated. This analysis was also made with larger step sizes $\left(20^{\circ}\right.$ and $\left.30^{\circ}\right)$ for the sampling of the four $\phi$ and $\psi$ dihedral angles, and also without the relaxation stage using Amber. Detailed results of this analysis are available as supporting information (Tables S8a and S10). For Cilengitide, with a grid search of $10^{\circ}$, EGSCyP ran for 188 CPU-hours. This time is divided by two without the Amber relaxation, which takes half of the exploration. The side chain placement is the second most time-consuming step, with more than $20 \%$ of the computing time. The backbone sampling (grid search + IK call) takes only less than $30 \%$ of the total 
run time. We should also note that we used a preliminary implementation of EGSCyP, non optimized, and running on one single core. The parallelization of of EGSCyP can be trivially achieved and would significantly speed up this approach. In this work, we performed an exhaustive exploration using a small step size of $10^{\circ}$ for the grid search sampling. However, a larger step size significantly decreases the simulation time (divided by 18 for $20^{\circ}$ and by 68 for $30^{\circ}$ ). This can be interesting for a more efficient exploration applying a multi-resolution strategy (this point is discussed below in the next subsection).

\section{Validation on Cilengitide}

\section{Comparison of Ramachandran diagrams}

The energy landscapes obtained with EGSCyP and REMD simulations were first compared using Ramachandran diagrams for each peptide and method. In Figure 3, the first two columns correspond to the conformations obtained by EGSCyP for the peptides RGDFV and RGDfV.

The last two columns correspond to the maps for Cilengitide (RGDfV') with the results obtained by the two methods: EGSCyP on the third column and REMD on the last column. Each line corresponds to one of the five residues of the cyclic pentapeptide. The diagrams corresponding to the comparison between EGSCyP and REMD for RGDFV and RGDfV peptides are available as supporting information in Figure S5.

For the EGSCyP method, the potential energy of the whole peptide was projected on the diagrams for each residue as a function of the $\phi$ and $\psi$ dihedral angles. This energy was computed in $\mathrm{kcal} / \mathrm{mol}$ with amberff96 force field at the last step of the relaxation with Sander. More precisely, a specific $\phi, \psi$ pair (within the grid spacing of 10 degrees), at a given residue position, is shared by a large number of conformations of the whole peptide, having a large distribution in energies. From each of these distributions, we only plotted the minimum energy value for this $\phi, \psi$ pair. The maps show that the exploration is quite complete, even including conformations of high energy (in blue). The white areas in Figure 3 correspond 


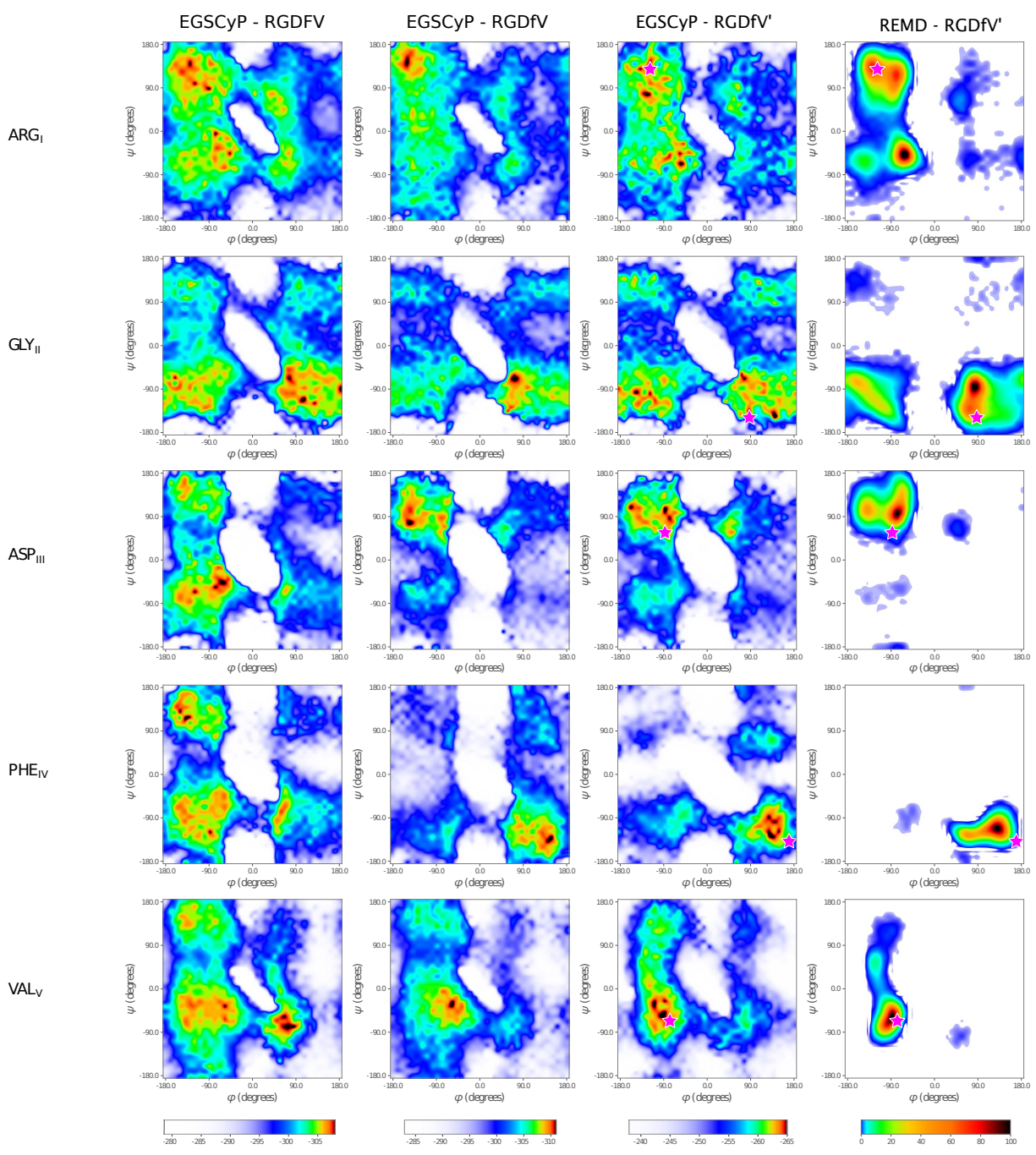

Figure 3: Ramachandran plots presenting the results obtained with the EGSCyP method for the RGDFV, RGDfV and RGDfV' peptides (three left columns) and the REMD simulations for RGDfV' (right column). The diagrams were created using the matplotlib python library. ${ }^{74}$ For EGSCyP, the energy landscape was projected as a function of the dihedral angles $\phi$ and $\psi$ for each residue (on each line). The color code corresponds to the minimal potential energy in $\mathrm{kcal} / \mathrm{mol}$ of the whole peptide associated to each combination of $\phi$ $\psi$ angles. Energy minima are represented in black/red, while areas with no conformation are in white, and areas of high energy are in blue. For REMD, the normalized frequency

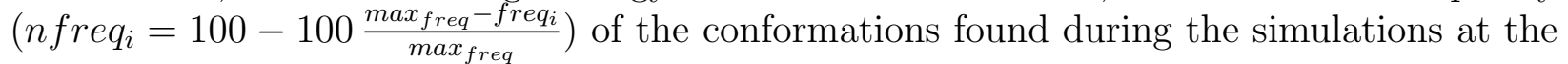
lowest temperature replica is projected as a function of $\phi$ and $\psi$. The color code corresponds to this normalized frequency, with the maximal frequency in black and a null frequency in white. The pink stars represent the values of the dihedral angles for the crystallized Cilengitide (sequence: RGDfV', PDB ID: 1L5G) in complex with integrin. 
either to (1) the absence of conformations because of the absence of solution at the IK solver step or of atomic collisions in the proposed structures; (2) conformations of very high energy. Although the global energy minimum is the same for all the five maps, the range of energies significantly varies. So, for a better understanding of the diagrams, we considered an energy threshold at $-235 \mathrm{kcal} / \mathrm{mol}$. This threshold was intended to be common to the five positions of the peptide, and calculated as follows: for each Ramachandran plot, the mean and the standard deviation of the projected energy values were summed. Then, the global energy threshold was defined as the maximum of these five sums. It provides an identical energy scale for all positions that explains the major part of each energy distribution and neglects out-layers.

Coverage percentage: We defined the coverage of the diagrams as the percentage of $\phi$ $\psi$ pairs, within the grid spacing of $10^{\circ}$, for which at least one conformation was found by EGSCyP. Here the ARG and VAL residues have been chosen as exhaustively sampled residues, with $97 \%$ and $98 \%$ of coverage level, while the other three are sampled by IK, with coverage levels of $86 \%$ to $93 \%$, confirming the fact that exhaustive sampling produces a larger coverage of the diagrams.

One may note that the coverage in the diagrams of Figure 3 seems lower due to the energy threshold and to the color gradient from blue to white. Details about the coverage percentage are available as supporting information in Table S5. This result shows again the completeness of the exploration. For REMD simulations, the normalized frequency of the observed conformations during the simulation was projected on the $\phi$ and $\psi$ axes for each residue. The coverage percentages are this time much lower, varying from $14 \%$ of coverage for the D-phenylalanine diagram up to $49 \%$ for the arginine diagram. This result is not totally surprising because REMD simulations tend to favor low energy areas and can have some difficulties to cross high energy barriers. In order to evaluate the performance of EGSCyP, we compared the coverage of the Ramachandran plots of Cilengitide for the two methods. For all residues, less than $1 \%$ of the areas in the diagrams are visited by REMD, 
but not by EGSCyP. In other words, $99 \%$ of the conformations in the Ramachandran plots produced by REMD are also sampled by EGSCyP. On the contrary, EGSCyP samples more conformations than REMD: the fraction of the map explored only by EGSCyP varies from $48 \%$ for glycine up to $79 \%$ for valine. This result supports again the good performance of our approach to exhaustively describe the conformational landscape.

Map similarities: A visual comparison of the maps clearly indicates that the lowestenergy basins for EGSCyP (in red and black, in the third column of Figure 3) correspond quite well to the high-population areas of the REMD simulations (in red and black too, in the fourth column of Figure 3). Areas with few (or no) conformations found during the REMD simulations, in blue (or white, respectively), correspond essentially to high-energy areas sampled by EGSCyP. Thoroughly analyzing the diagrams, some minor differences between the two methods can be observed. REMD simulations produced only one black region per residue (corresponding to the maxima of frequency) and one or two other regions in red/orange, while EGSCyP resulted in several black points (corresponding to several energy minima). Note however that both maps display different quantities: while the EGSCyP method is based on the potential energy, frequencies derived from the REMD trajectories reflect the total free energy of the system, including the entropic contribution. The difference between the potential energy of the black and red/orange areas is very low with EGSCyP, typically $2 \mathrm{kcal} / \mathrm{mol}$. This explains why the valine and phenylalanine diagrams present three black dots in EGSCyP but only one in REMD. These three points in EGSCyP maps correspond to the same energy basin in REMD maps.

Run time and completeness of the exploration: Some comparisons about the run time and the completeness of the exploration were made for EGSCyP and REMD. For EGSCyP, simulations were made with step sizes of $10^{\circ}$ (with and without the relaxation using Amber), $20^{\circ}$ and $30^{\circ}$. For REMD, the $2.4 \mu$ s simulation was cut into smaller segments $(600 \mathrm{~ns}, 240 \mathrm{~ns}$, $24 \mathrm{~ns})$. For both methods, percentages of coverage and projected landscapes on Ramachandran diagrams are available as supporting information (see Figures S9 and S10; Tables S8 
to S10). An interesting point is that the two methods do not show the same variation of the exploration as the run time decreases. On the one hand, REMD simulations, for any part of the split trajectory, show quite similar high frequency areas of the projected landscapes on the maps. Nevertheless, the percentage of coverage decreases with shorter simulation times. On the other hand, EGSCyP shows landscapes with higher energy with a $10^{\circ}$ step, but without Amber or with a $20^{\circ}$ step. In the case of a $30^{\circ}$ step, the energy minima are located in different areas, that may be due to the resolution of the simulation. The percentages of coverage for the three residues sampled by IK still stay very high (at least $65 \%$ for $30^{\circ}$ ). Nevertheless, these percentages decrease significantly for the two exhaustively sampled residues. This result was expected as the number of combination tested is drastically reduced (at $16 \%$ at $30^{\circ}$ step) when increasing the step size. Despite the degraded quality, the main energy basins can still be identified even when performing a very coarse exploration. Therefore, to escape from this sampling effect, it would be possible to implement a multi-resolution version of EGSCyP: first, a fast low-resolution exploration (with a step size of $20^{\circ}$ or $30^{\circ}$ ) is performed to identify the energy basins, followed by a more accurate exploration inside these areas. The overall procedure could easily be parallelized, which would allow a significant speed up.

\section{Comparison of energy minima conformations}

Clustering: We applied our clustering approach to the results obtained by EGSCyP and REMD simulations for Cilengitide (RGDfV') in order to compare the energy minima conformations. As a distance metric, we used RMSD based on the $\phi-\psi$ dihedral angles. In general, this provides better results than $\mathrm{C}_{\alpha}$-RMSD for clustering conformations of peptides. In particular, angular RMSD is very useful to identify peptide-plane flips: ${ }^{75}$ a large amplitude rotation around the peptide plane affecting the values of the $\psi_{i}$ and the $\phi_{i+1}$ dihedral angles. Indeed, the variations of these two angles mutually compensate, such that the RMSD computed on the $\alpha$ carbons does not show any fluctuation. ${ }^{76}$ However, such a perturbation 
can affect the conformation of the peptide, in particular by restraining the side chains conformation. The distance threshold for clustering was set to $60^{\circ}$, which allows distinguishing important peptide-plane flips.

We obtained 120 clusters with EGSCyP against 12 clusters with REMD simulations for Cilengitide. This huge difference is once again not surprising and is consistent with the previous results (percentage coverage of Ramachandran diagrams), demonstrating the exhaustiveness of the EGSCyP exploration. The diversity of the clusters are presented as supporting information (Figures S6 and S7).

With REMD, the first and most populated cluster represents $57 \%$ of the conformations found during the simulation. The second cluster represents $42 \%$. This means that $99 \%$ of the conformations are described by these two clusters. We extracted from the trajectory the two structures representing the centers of these clusters and minimized them with Sander. We will call the minimized conformations $\min _{1}^{\text {REMD }}$ and $\min _{2}^{\text {REMD }}$ in the following. Their energies are very similar: -267.6 kcal/mol and -267.9 kcal/mol, respectively. Representative conformations of the two first clusters obtained with EGSCyP were also locally minimized without any restraint. The energies of these two minima, called $\min _{1}^{\text {EGSCyP }}$ and $\min _{2}^{\text {EGSCyP }}$, are $-267.3 \mathrm{kcal} / \mathrm{mol}$ and $-267.4 \mathrm{kcal} / \mathrm{mol}$, respectively. As for the two main minima obtained from REMD, the energy values of the minima for EGSCyP are extremely similar. This is in accordance with the fact that peptides, unlike the proteins, present several energetically equivalent minima. Note that the energy values for the minima obtained by the two methods are also very close. Figure 4 shows the superimposed structures of these four minima: in Figure $4 \mathrm{a}, \min _{1}^{\mathrm{REMD}}$ (in green) and $\min _{1}^{\mathrm{EGSCyP}}$ (in beige); in Figure $4 \mathrm{~b}, \min _{2}^{\mathrm{REMD}}$ (in pink) and $\min _{2}^{\mathrm{EGSCyP}}$ (in blue).

The backbones show an important similarity between both methods: $\min _{1}^{\text {REMD }}$ and $\min _{1}^{\text {EGSCyP }}$ have the same distribution of dihedral angles with an angular RMSD of $32^{\circ}$ between them. The second clusters, $\min _{2}^{\text {REMD }}$ and $\min _{2}^{\text {EGSCyP }}$, also correspond to each other with a RMSD of $32^{\circ}$. The values of the dihedral angles are presented as supporting infor- 


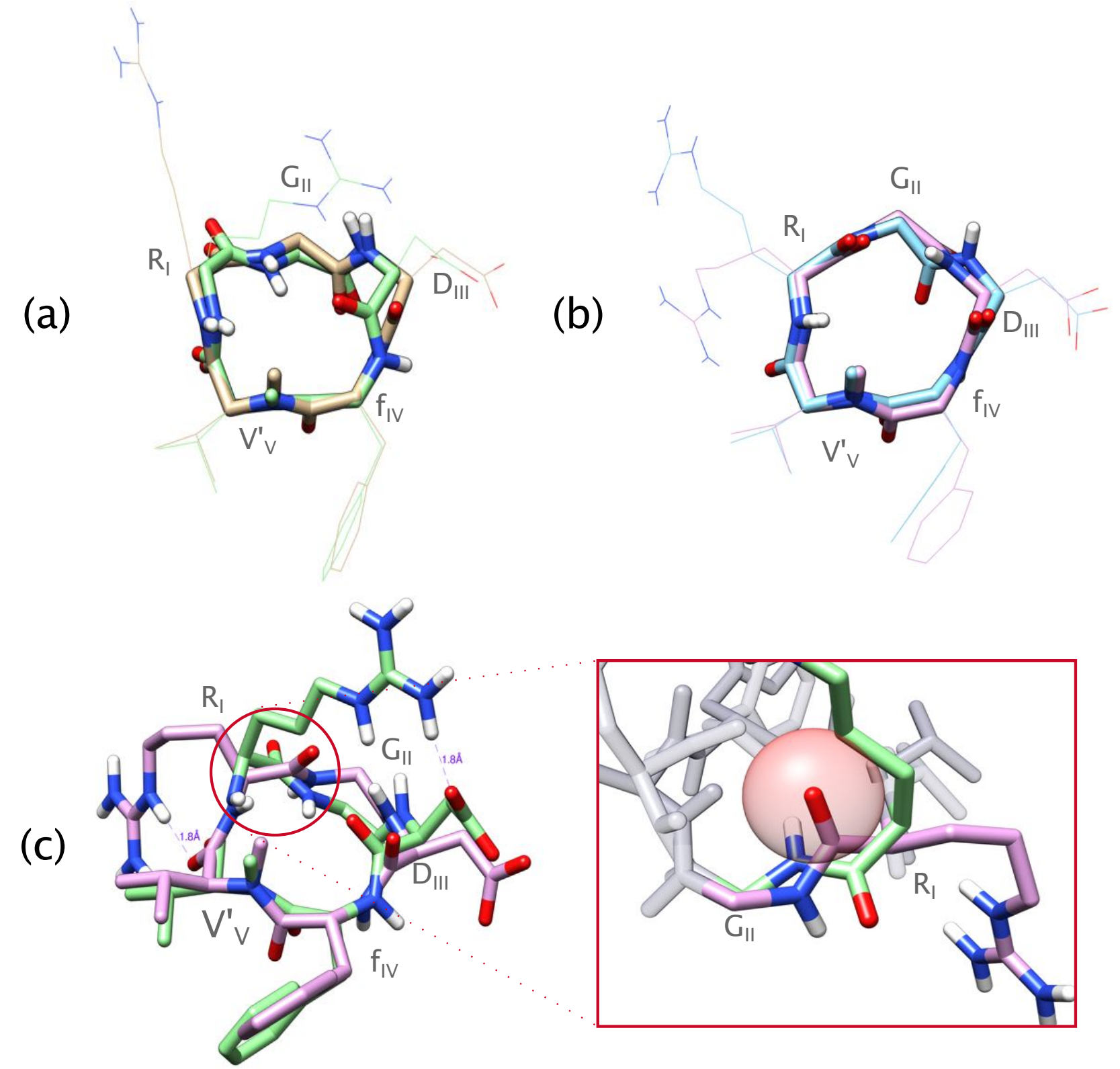

Figure 4: Lowest energy conformations obtained for Cilengitide (RGDfV') with EGSCyP and REMD. (a) Superimposition of the structures of $\min _{1}^{\text {REMD }}$ (in green) and $\min _{1}^{\text {EGSCyP }}$ (in beige). RMSD on dihedral angles between both structures equals $32^{\circ}$. (b) Superimposition of the structures of $\min _{2}^{\text {REMD }}$ (in pink) and $\min _{2}^{\text {EGSCyP }}$ (in blue). RMSD between the two structure equals $32^{\circ}$. For (a) and (b) the side chains are represented in lines for better visibility. (c) Superimposition of the structures of $\min _{1}^{\text {REMD }}$ (in green) and $\min _{2}^{\text {REMD }}$ (in pink). The purple dashed lines represent the measurement of the distance between two atoms. The major difference between the two clusters is a peptide flip between the arginine and glycine surround in red. On the right part of the figure, a zoom was made on the flip from the other side for a better visibility. The red sphere represents the van Der Waals radius of the carbonyl oxygen atom from $\min _{2}^{\mathrm{REMD}}$. 
mation (Table S6). The most significant difference between the two groups (Figure 4a and Figure 4b) corresponds to a peptide-plane flip between $\psi_{I}$ and $\phi_{I I}$ angles. This difference between the two main minima is illustrated in Figure 4c. The structures of $\min _{1}^{\text {REMD }}$ and $\min _{2}^{\text {REMD }}$ are represented with carbon atoms in green and pink respectively. The flip explaining the difference is surrounded in red. It impacts the position of the arginine side chain. Indeed, in $\min _{1}^{R E M D}$, the side chain can fold and create a hydrogen bond with the side chain (in green) of the aspartate. Considering $\min _{2}^{R E M D}$, the side chain (in pink) is oriented more outwards and makes a hydrogen bond with the oxygen of the valine carbonyl group. The comparison of the two conformations shows that the flip leads to a tilt of the carbonyl group of the glycine visible on the onset of Figure 4c. This prevents the arginine side chain of the second minimum to come closer to the cycle and interact with the aspartate side chain. Indeed, the orientation of the oxygen atom would create a collision if the side chain would attempt to get closer (see van Der Waals radius of the carbonyl oxygen atom in the zoom of Figure 4c). Therefore, the tilt has an impact on the structure, constraining the arginine side chain (even if it does not mean that all the structures from the cluster corresponding to $\min _{1}^{\mathrm{REMD}}$ have this side chain orientation).

Comparison with crystallographic structure: Results provided by the two conformational exploration methods were compared to the X-Ray structure of Cilengitide bound to integrin (PDB code: 1L5G). The dihedral angles values of this X-Ray structure were projected on all the RGDfV' Ramachandran diagrams. They are indicated with a pink star in the third and forth columns of Figure 3. We can observe that this experimental structure is close to energy minima (in black). The RMSD on dihedral angles was computed between this structure and the clustered minima structures from the two methods (see section above). The $\min _{2}^{\text {EGSCyP }}$ minimum is the closest one from the X-Ray structure, with RMSD equal to $14^{\circ}$. The RMSD for $\min _{2}^{\text {REMD }}$ is $35^{\circ}$. The first minima $\left(\min _{1}^{\text {EGSCyP }}\right.$ and $\min _{1}^{\text {REMD }}$ ) are more distant to the experimental structure, with RMSD around $75^{\circ}$ for both methods. In Figure 5 , the experimental structure (in purple) is superimposed to $\min _{2}^{\mathrm{EGSCyP}}$ (in blue) in order to 
show the good similarity of the two conformations. However, $\min _{2}^{\text {EGSCyP }}$ and $\min _{2}^{\text {REMD }}$ are both very close to the crystallographic one. Three hypotheses can explain why the result of EGSCyP better matches the bound state compared to REMD.

(1) EGSCyP explores a larger landscape than REMD, due the algorithm itself and as measured by the percentage of coverage, so that it probably finds one conformation closer to the crystallographic one with a lower energy.

(2) The clustering method induces a bias. It may be possible that among all the REMD conformations of the cluster corresponding to this energy minimum, other conformations are closer to the crystallographic structure but with an energy slightly higher, explaining that these were not chosen as the center of the cluster.

(3) One can notice that the experimental peptide is bound to the protein (superimpositions with the protein complex are available in Figure S13 as supporting information). This bound conformation may not be the global energy minimum for the free peptide, but a close conformation that is slightly rearranged when binding. We also compared the energy minima with the free structure of Cilengitide obtained from NMR experiments ${ }^{77}$ (data kindly provided by Pr Horst Kessler). The free and bound conformations are close to each other with a backbone RMSD of $29^{\circ}$ when superimposed (Figure S12). For the REMD method, $\min _{2}^{R E M D}$ is slightly closer to the free experimental conformation $\left(\mathrm{RMSD}=23^{\circ}\right.$ ) than to the bound one $\left(\mathrm{RMSD}=35^{\circ}\right)$. The RMSD distance between $\min _{2}^{\mathrm{EGSCyP}}$ and the free NMR structure of Cilengitide is very similar: $24^{\circ}$.

\section{Side chain placement}

Figure 6 represents the projections of the energy landscape of the RGDFV peptide as a function of the $\chi_{1}$ and $\chi_{2}$ dihedral angles ${ }^{78}$ for the arginine, aspartate and phenylalanine residues.

The first column corresponds to the conformations obtained with the EGSCyP using SCRWL4 for the side chain positioning. The second column corresponds to the EGSCyP 


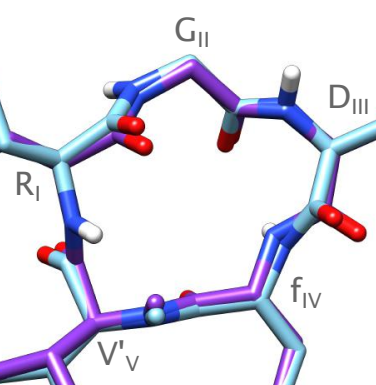

Figure 5: Structure of Cilengitide (RGDfV'). Comparison between the crystallographic structure (PDB ID: 1L5G, in purple) and the closest energy minimum found by EGSCyP $\left(\min _{2}^{\text {EGSCyP }}\right.$ in blue). RMSD of the dihedral angles between them is $14^{\circ}$.

with the alternation of $\mathrm{BH}$ and local minimization, referred to as $\mathrm{BH} / \mathrm{MC}$ in the following. The last column corresponds to the conformations from the REMD simulations. The color code corresponds to the potential energy of the whole peptide for the EGSCyP and to the normalized frequency of the conformations found during the simulations at the lowest temperature replica for the REMD simulations.

The $\chi_{1}-\chi_{2}$ maps show that there is a striking difference between the two methods for the side chain placement. With SCWRL4, the covered space is very small compared to the one obtained with $\mathrm{BH} / \mathrm{MC}$ or the REMD simulation. Actually, SCWRL4 covered $8 \%$ to $16 \%$ of the diagrams while the $\mathrm{BH} / \mathrm{MC}$ covered between $54 \%$ and $69 \%$. Less than $1 \%$ of the space is covered only by SCWRL4 and not by BH/MC meaning that the space explored by SCWRL4 is almost totally included in the one explored by BH/MC. Regarding the quality of the obtained conformations, the energy minima obtained with SCRWL4 are not localized at the same place as the frequency maxima obtained by REMD. For the arginine residue, the minima are rather close to the frequency maxima for the arginine obtained by REMD, but for the aspartate and phenylalanine, they are not localized on the areas of 

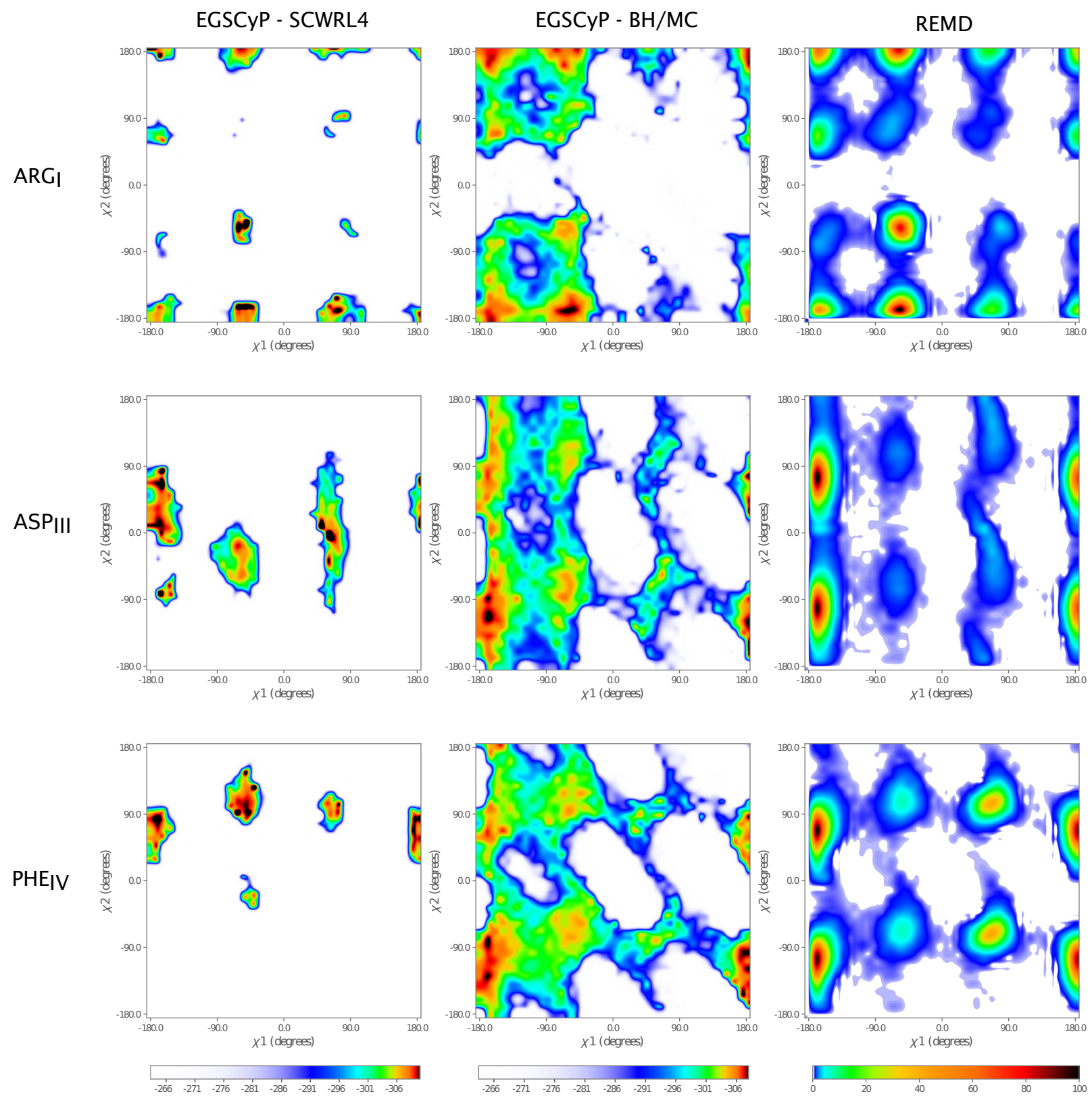

Figure 6: Comparison of the side chain conformational landscapes obtained for the RGDFV cyclic peptide with EGSCyP using SCWRL4 ${ }^{50}$ (left column), with EGSCyP and the alternation of $\mathrm{BH}$ and local minimization (middle column), and with the REMD simulations (right column). The conformational landscape was projected as a function of the $\chi_{1}-\chi_{2}$ dihedral angles of the side chains for ARG (first line), ASP (second line) and PHE (last line). The diagrams were created using the matplotlib python library. ${ }^{74}$ For EGSCyP, the color codes correspond to the minimal potential energy of the whole peptide associated to this combination of $\chi_{1}-\chi_{2}$. For the REMD simulations, the color code corresponds to the normalized frequency $\left(n f r e q_{i}=100-100 \frac{\max _{f_{r e q}-f_{r e q}} \text { max }}{\text { fareq }_{\text {freq }}}\right.$ ) of the conformations found during the simulations at the lowest temperature replica as a function of $\chi_{1}$ and $\chi_{2}$. 
high frequency of the REMD simulations. On the contrary, the $\mathrm{BH} / \mathrm{MC}$ sampling shows a good correspondence between its energy minima basins and the frequency maxima from the REMD simulations for the three residues of Figure 6. The percentage coverage for the REMD side chain placement is rather constant among the different residues (around 58\%) and the part of the coverage in common between the $\mathrm{BH} / \mathrm{MC}$ and $\mathrm{REMD}$ is about $50 \%$ of the diagrams. Actually, less than $10 \%$ of the coverage in the REMD diagrams are not covered by $\mathrm{BH} / \mathrm{MC}$. Therefore, this study confirms that the use of SCWRL4 is definitely not adapted for the case of small cyclic peptides. Moreover, the method cannot deal with chemical modifications. These results also validate the good performance of the alternation $\mathrm{BH} / \mathrm{MC}$ for side chain placement within EGSCyP. The $\chi_{1}-\chi_{2}$ diagrams for the other three peptides are available in supporting information (Figure S8). Similarly, they show a good accordance between $\mathrm{BH} / \mathrm{MC}$ and REMD coverage of the side chain maps (Table S7), in particular with the experimental structure of Cilengitide.

\section{Effects of the chemical modification on the energy landscape}

The exploration of the conformational landscape of the three RGD peptides (RGDFV, RGDfV and RGDfV'), was compared to analyze the influence of the chemical modifications, as it can be observed in Figure 3.

\section{D-residues}

The conformational landscape of the D-residue (noted as a lower case letter) changes compared to its L-enantiomer, as expected. The accepted conformations show some symmetry relative to the centers of the Ramachandran plots of Figure 3. There are three energy minima basins for the L-enantiomer, but only one for the D-enantiomer (black dots in the first and second columns of Figure 3). Nevertheless, the major basin for phenylalanine (upper left for the L-enantiomer) is conserved through symmetry in the D-enantiomer. Therefore, the landscape of the D-residue seems more restrained than the one of the L-enantiomer, confirm- 
ing the properties of the D-residues to locally constrain the conformation of the molecule. ${ }^{2}$ Actually, the percentage of coverage is quite similar between the two diagrams (about 93\%), but the potential energy is globally higher with the D-residue (blue areas).

The replacement of the $\mathrm{L}$ by the D-enantiomer of the phenylalanine has an impact on the landscapes of the whole peptide, extending to the second neighbors. This can be observed both on the level of the covered surface and the number of basins. The smaller number of basins within the peptide containing one L amino acid suggests a higher global constrained conformation when a non natural amino acid is included. The two direct neighbors are particularly impacted: for the downstream valine, one (around $\phi=70$ and $\psi=-70$ ) of the two energy minima basins vanishes and the area $\phi>0$ has conformations of higher energies. For the upstream aspartate, the basin goes from the lower part of the diagram $(\psi<0)$ to the upper part $(\psi>0)$ and the area with $\psi<0$ is of higher energies. For the second neighbor, differences can still be shown: the glycine energy basin from the lower left part of the diagram vanishes, the one from the lower right part is much less extensive, and the upper part of the diagram $(\psi>0)$ presents higher energies when the L-phenylalanine is used. For the arginine, the differences are the following: the energy basin from the lower part of the diagram disappears and the one from the upper part shrinks; the right part of the diagram also presents higher energies with the D-enantiomer. Therefore, these remarks confirm that the D-enantiomer does not only constrain the conformational landscape of the peptide, but also affects its direct and indirect neighbors.

\section{N-methylation}

The consequence of the addition of a N-methylation on valine can be evaluated by comparing the RGDfV and RGDfV' peptides (Figure 3, column 2 and 3). The coverage percentage of the landscape is quite constant, even in spite of the increasing number of collisions. Inspecting the diagram for each residue, one can make the following observations. 1) The presence of the methyl on the backbone shifts the energy towards higher values at the location of the 
modification, so it is consistent with some kind of destabilizing effect of this modification. 2) The central zone of the Ramachandran plot, highly unfavorable, is enlarged for the modified residue; this does not significantly affect the other residues. 3) The number of basins goes from one for the natural valine up to three for the modified one. 4) The diffusing effect of the methylation occurs on the landscape of arginine, the first neighbour in the cycle. One recovers the basins in the lower left of the ARG diagram that disappeared under the presence of the D-phenylalanine. Therefore, there is a sort of balance between the constraints introduced by the D-form and the methylation that increase the number of basins. Thus, one may hypothesize that the addition of the N-methyl offsets a part of the constraints brought by the D-enantiomer.

\section{Conclusions}

Small cyclic peptides present unique properties making them promising therapeutics drugs. The current difficulty to predict the structure and to design cyclic peptides could be greatly improved thanks to a better understanding of their whole conformational landscape. In this paper, we propose a method, called EGSCyP, for the exhaustive exploration of the energy landscape of cyclic pentapeptides possibly involving chemical modifications. We have shown the good performance of the method, which is based on a robotics approach and a multi-level representation of the peptide. The comparison of the results obtained for three cyclic pentapeptides with REMD simulations reveals the completeness and consistence of our approach. Also, the comparison with the experimentally-determined structure of Cilengitide bound to the integrin complex demonstrates the predictive capabilities of the method. Moreover, we have demonstrated the effectiveness of the alternation of $\mathrm{BH} /$ local minimization for the sampling of the side chains conformations, whereas SCWRL4 fails to correctly position the side chains of small cyclic peptides. Finally, this approach clearly shows the effect on the conformational landscape of the D-residue that constrains the landscape 
and the N-methylation that also modulates it.

In the short future, we will apply EGSCyP to a larger dataset, including all the available short head to tail cyclic peptide experimental structures, in order to more robustly evaluate the efficiency and accuracy of our approach. Other force fields could be used within our approach. Indeed, the accuracy of the energy computation for EGSCyP as well as REMD simulations depend on the force field used. Thus, it would be interesting to test other force fields, and in particular optimized force fields for cyclic peptides. For instance, the RSFF1 and RSFF2 force fields have shown good performance on cyclic peptides. ${ }^{79}$ However, progress is still needed, since it has been shown that these force fields are for the moment not well suited to the use of non natural residues like the N-methylated ones ${ }^{80}$ neither for implicit solvent. We also plan to investigate conformational changes using the connectivity graph built during the exploration. The better understanding of the conformational properties of small cyclic peptides may be used to develop more suitable methods for structure prediction and design. Finally mention that we intend to extend the approach to larger cyclic peptides. Obviously, a systematic, grid-based exploration as described in this paper has limits in the length of the peptide candidates due to the combinatorial explosion. However, the proposed multi-level modeling approach can be exploited within stochastic exploration-optimization methods, such as variants of $\mathrm{BH}$, able to provide a global picture of the conformational landscape.

\section{Availability}

We are developing a software package called MoMA (for Molecular Motion Algorithms) including modeling tools and algorithms to sample conformations and transition paths of biomolecules. The methods presented in this paper have been implemented in this software package. The open-source code, in $\mathrm{C}++$, is not available yet. Nevertheless, binaries can be provided upon request. The IK solver used in this work can also be provided as a stand-alone 
$\mathrm{C}++$ library.

\section{Acknowledgement}

This work was performed using HPC resources from GENCI-CINES (Grant 2016-c2016077641). CALMIP is gratefully acknowledged for the access to the super-computer EOS (allocation 2016-P16032) for developing the EGSCyP method. We also thank Dr Michele Lazzeri for letting us using his cluster, and Dr Guillaume Postic for valuable discussions and advices. Finally, we express our sincere thanks to Pr Horst Kessler for giving us NMR structure of Cilengitide.

\section{Supporting Information Available}

Figures S1-S13; Tables S1- S10.

\section{References}

(1) Eustache, S.; Leprince, J.; Tufféry, P. Progress with Peptide Scanning to study Structure-Activity Relationships: the Implications for Drug Discovery. Expert Opin. Drug Discovery 2016, 11, 771-784.

(2) Gongora-Benitez, M.; Tulla-Puche, J.; Albericio, F. Multifaceted Roles of Disulfide Bonds. Peptides as Therapeutics. Chem. Rev. (Washington, DC, U. S.) 2013, 114, 901-926.

(3) Conibear, A. C.; Chaousis, S.; Durek, T.; Johan Rosengren, K.; Craik, D. J.; Schroeder, C. I. Approaches to the Stabilization of Bioactive Epitopes by Grafting and Peptide Cyclization. Pept. Sci. 2016, 106, 89-100. 
(4) Allen, S. E.; Dokholyan, N. V.; Bowers, A. A. Dynamic Docking of Conformationally Constrained Macrocycles: Methods and Applications. ACS Chem. Biol. 2015, 11, 1024.

(5) Gao, M.; Cheng, K.; Yin, H. Targeting Protein- Protein Interfaces using Macrocyclic Peptides. Pept. Sci. 2015, 104, 310-316.

(6) Driggers, E. M.; Hale, S. P.; Lee, J.; Terrett, N. K. The Exploration of Macrocycles for Drug Discoveryan Underexploited Structural Class. Nat. Rev. Drug Discovery 2008, 7, 608-625.

(7) Craik, D. J.; Fairlie, D. P.; Liras, S.; Price, D. The Future of Peptide-based Drugs. Chem. Biol. Drug Des. 2013, 81, 136-147.

(8) Bhardwaj, G.; Mulligan, V. K.; Bahl, C. D.; Gilmore, J. M.; Harvey, P. J.; Cheneval, O.; Buchko, G. W.; Pulavarti, S. V.; Kaas, Q.; Eletsky, A.; Huang, P.-S.; Johnsen, W. A.; Greisen, P. J.; Rocklin, G. J.; Song, Y.; Linsky, T. W.; Watkins, A.; Rettie, S. A.; Xu, X.; Carter, L. P.; Bonneau, R.; Olson, J. M.; Coutsias, E.; Correnti, C. E.; Szyperski, T.; Craik, D. J.; Baker, D. Accurate de novo Design of Hyperstable Constrained Peptides. Nature 2016, 538, 329-335.

(9) Vanhee, P.; van der Sloot, A. M.; Verschueren, E.; Serrano, L.; Rousseau, F.; Schymkowitz, J. Computational Design of Peptide Ligands. Trends Biotechnol. 2011, 29, 231-239.

(10) Wells, J. A.; McClendon, C. L. Reaching for High-Hanging Fruit in Drug Discovery at Protein-Protein Interfaces. Nature 2007, 450, 1001-1009.

(11) McHugh, S. M.; Rogers, J. R.; Yu, H.; Lin, Y.-S. Insights into How Cyclic Peptides Switch Conformations. J. Chem. Theory Comput. 2016, 12, 2480-2488. 
(12) Robinson, J. A.; DeMarco, S.; Gombert, F.; Moehle, K.; Obrecht, D. The Design, Structures and Therapeutic Potential of Protein Epitope Mimetics. Drug discovery today 2008, 13, 944-951.

(13) Olmez, E. O.; Akbulut, B. S. Binding Protein; InTech, 2012.

(14) Vlieghe, P.; Lisowski, V.; Martinez, J.; Khrestchatisky, M. Synthetic Therapeutic Peptides: Science and Market. Drug discovery today 2010, 15, 40-56.

(15) Hill, T. A.; Shepherd, N. E.; Diness, F.; Fairlie, D. P. Constraining Cyclic Peptides to Mimic Protein Structure Motifs. Angew. Chem., Int. Ed. 2014, 53, 13020-13041.

(16) Oakley, M. T.; Johnston, R. L. Exploring the Energy Landscapes of Cyclic Tetrapeptides with Discrete Path Sampling. J. Chem. Theory Comput. 2012, 9, 650-657.

(17) Chatterjee, J.; Gilon, C.; Hoffman, A.; Kessler, H. N-methylation of Peptides: a New Perspective in Medicinal Chemistry. Acc. Chem. Res. 2008, 41, 1331-1342.

(18) Wójcik, P.; Berlicki, Ł. Peptide-based Inhibitors of Protein-Protein Interactions. Bioorg. Med. Chem. Lett. 2016, 26, 707-713.

(19) Kamenik, A. S.; Lessel, U.; Fuchs, J. E.; Fox, T.; Liedl, K. R. Peptidic MacrocyclesConformational Sampling and Thermodynamic Characterization. J. Chem. Inf. Model. 2018, 58, 982-992.

(20) Mas-Moruno, C.; Beck, J. G.; Doedens, L.; Frank, A. O.; Marinelli, L.; Cosconati, S.; Novellino, E.; Kessler, H. Increasing $\alpha \mathrm{v} \beta 3$ Selectivity of the Anti-Angiogenic Drug Cilengitide by N-Methylation. Angew. Chem., Int. Ed. 2011, 50, 9496-9500.

(21) Thevenet, P.; Shen, Y.; Maupetit, J.; Guyon, F.; Derreumaux, P.; Tuffery, P. PEPFOLD: an Updated de novo Structure Prediction Server for both Linear and Disulfide Bonded Cyclic Peptides. Nucleic Acids Res. 2012, 40, W288-W293. 
(22) Shen, Y.; Maupetit, J.; Derreumaux, P.; Tuffery, P. Improved PEP-FOLD Approach for Peptide and Miniprotein Structure Prediction. J. Chem. Theory Comput. 2014, 10, $4745-4758$.

(23) Singh, S.; Singh, H.; Tuknait, A.; Chaudhary, K.; Singh, B.; Kumaran, S.; Raghava, G. P. PEPstrMOD: Structure Prediction of Peptides Containing Natural, Non-Natural and Modified Residues. Biol. Direct 2015, 10, 73-73.

(24) Zhang, Y. I-TASSER Server for Protein 3D Structure Prediction. BMC Bioinf. 2008, 9, 40-40.

(25) Beaufays, J.; Lins, L.; Thomas, A.; Brasseur, R. In Silico Predictions of 3D Structures of Linear and Cyclic Peptides with Natural and non-Proteinogenic Residues. J. Pept. Sci. 2012, 18, 17-24.

(26) Hosseinzadeh, P.; Bhardwaj, G.; Mulligan, V. K.; Shortridge, M. D.; Craven, T. W.; Pardo-Avila, F.; Rettie, S. A.; Kim, D. E.; Silva, D.-A.; Ibrahim, Y. M.; Webb, I. K.; Cort, J. R.; Adkins, J. N.; Varani, G.; Baker, D. Comprehensive Computational Design of Ordered Peptide Macrocycles. Science 2017, 358, 1461-1466.

(27) Ramakrishnan, C.; Paul, P.; Ramnarayan, K. Cyclic PeptidesSmall and Big and their Conformational Aspects. J. Biosci. 1985, 8, 239-251.

(28) Ramachandran, G. t.; Sasisekharan, V. Advances in protein chemistry; Elsevier, 1968; Vol. 23; pp 283-437.

(29) McHugh, S. M.; Rogers, J. R.; Solomon, S. A.; Yu, H.; Lin, Y.-S. Computational Methods to Design Cyclic Peptides. Curr. Opin. Chem. Biol. 2016, 34, 95-102.

(30) Paissoni, C.; Ghitti, M.; Belvisi, L.; Spitaleri, A.; Musco, G. Metadynamics Simulations Rationalise the Conformational Effects Induced by N-Methylation of RGD Cyclic Hexapeptides. Chem. - Eur. J. 2015, 21, 14165-14170. 
(31) Karplus, M.; McCammon, J. A. Molecular Dynamics Simulations of Biomolecules. Nat. Struct. Mol. Biol. 2002, 9, 646-646.

(32) Daura, X.; Gademann, K.; Jaun, B.; Seebach, D.; van Gunsteren, W. F.; Mark, A. E. Peptide Folding: when Simulation meets Experiment. Angew. Chem., Int. Ed. 1999, 38, 236-240.

(33) Yu, H.; Lin, Y.-S. Toward Structure Prediction of Cyclic Peptides. Phys. Chem. Chem. Phys. 2015, 17, 4210-4219.

(34) Slough, D. P.; McHugh, S. M.; Lin, Y.-S. Understanding and Designing Head-to-Tail Cyclic Peptides. Biopolymers 2018, e23113-e23113.

(35) Wakefield, A. E.; Wuest, W. M.; Voelz, V. A. Molecular Simulation of Conformational Pre-Organization in Cyclic RGD Peptides. J. Chem. Inf. Model. 2015, 55, 806-813.

(36) Go, N.; Scheraga, H. A. Ring Closure and Local Conformational Deformations of Chain Molecules. Macromolecules 1970, 3, 178-187.

(37) Wu, M. G.; Deem, M. W. Analytical Rebridging Monte Carlo: Application to cis/trans Isomerization in Proline-Containing, Cyclic Peptides. J. Chem. Phys. 1999, 111, 66256632.

(38) Coutsias, E. A.; Seok, C.; Jacobson, M. P.; Dill, K. A. A Kinematic View of Loop Closure. J. Comput. Chem. 2004, 25, 510-528.

(39) Siciliano, B., Khatib, O., Eds. Springer Handbook of Robotics; Springer: New York, 2008.

(40) Wermuth, J.; Goodman, S.; Jonczyk, A.; Kessler, H. Stereoisomerism and Biological Activity of the Selective and Superactive $\alpha \mathrm{v} \beta 3$ Integrin Inhibitor Cyclo (-RGDfV-) and its Retro-Inverso Peptide. J. Am. Chem. Soc. 1997, 119, 1328-1335. 
(41) Dechantsreiter, M. A.; Planker, E.; Mathä, B.; Lohof, E.; Hölzemann, G.; Jonczyk, A.; Goodman, S. L.; Kessler, H. N-Methylated Cyclic RGD Peptides as Highly Active and Selective $\alpha \mathrm{v} \beta 3$ Integrin Antagonists. J. Med. Chem. 1999, 42, 3033-3040.

(42) Mas-Moruno, C.; Rechenmacher, F.; Kessler, H. Cilengitide: the First Anti-Angiogenic Small Molecule Drug Candidate. Design, Synthesis and Clinical Evaluation. AntiCancer Agents Med. Chem. 2010, 10, 753-768.

(43) Mas-Moruno, C. Peptides and Proteins as Biomaterials for Tissue Regeneration and Repair; Elsevier, 2018; pp 73-100.

(44) Schlick, T. Molecular Modeling and Simulation: an Interdisciplinary Guide; Springer Science \& Business Media: Philadelphia, 2010; Vol. 21.

(45) Scott, R. A.; Scheraga, H. A. Conformational Analysis of Macromolecules. III. Helical Structures of Polyglycine and Poly-L-Alanine. J. Chem. Phys. 1966, 45, 2091-2101.

(46) Parsons, D.; Canny, J. Geometric Problems in Molecular Biology and Robotics. Proc. - Int. Conf. Intell. Syst. Mol. Biol., 2th 1994, 322-330.

(47) Al-Bluwi, I.; Siméon, T.; Cortés, J. Motion Planning Algorithms for Molecular Simulations: A Survey. Comput. Sci. Rev. 2012, 6, 125-143.

(48) Shehu, A.; Plaku, E. A Survey of Computational Treatments of Biomolecules by Robotics-Inspired Methods Modeling Equilibrium Structure and Dynamic. J. Artif. Intell. Res. 2016, 5\%, 509-572.

(49) Bondi, A. van der Waals Volumes and Radii. J. Phys. Chem. 1964, 68, 441-451.

(50) Krivov, G. G.; Shapovalov, M. V.; Dunbrack, R. L. Improved Prediction of Protein Side-Chain Conformations with SCWRL4. Proteins: Struct., Funct., Bioinf. 2009, r7, $778-795$. 
(51) Wales, D. J.; Doye, J. P. Global Optimization by Basin-Hopping and the Lowest Energy Structures of Lennard-Jones Clusters Containing up to 110 Atoms. J. Phys. Chem. A 1997, 101, 5111-5116.

(52) Case, D. A.; Betz, R.; Cerutti, D.; Cheatham III, T.; Darden, T.; Duke, R.; Giese, T.; Gohlke, H.; Goetz, A.; Homeyer, N.; Izadi, S.; Janowski, P.; Kaus, J.; Kovalenko, A.; Lee, T.; LeGrand, S.; Li, P.; Lin, C.; Luchko, T.; Luo, R.; Madej, B.; Mermelstein, D.; Merz, K.; Monard, G.; Nguyen, H.; Nguyen, H.; Omelyan, I.; Onufriev, A.; Roe, D.; Roitberg, A.; Sagui, C.; Simmerling, C.; Botello-Smith, W.; Swails, J.; Walker, R.; Wang, J.; Wolf, R.; Wu, X.; Xiao, L.; Kollman, P. AMBER 2016. UCSF 2016, 1, 3-3.

(53) Kollman, P. A. Advances and Continuing Challenges in Achieving Realistic and Predictive Simulations of the Properties of Organic and Biological Molecules. Acc. Chem. Res. 1996, 29, 461-469.

(54) Shell, M. S.; Ritterson, R.; Dill, K. A. A Test on Peptide Stability of AMBER Force Fields with Implicit Solvation. J. Phys. Chem. B 2008, 112, 6878-6886.

(55) Craig, J. J. Introduction to Robotics; Addison-Wesley: upper Saddle River, New Jersey, 1989.

(56) Renaud, M. Current Advances in Mechanical Design and Production VII. 2000.

(57) Renaud, M. Calcul des Modeles Géométriques Inverses des Robots Manipulateurs 6R. 2006.

(58) Lee, H.-Y.; Liang, C.-G. A New Vector Theory for the Analysis of Spatial Mechanisms. Mech. Mach. Theory 1988, 23, 209-217.

(59) Lee, H.-Y.; Liang, C.-G. Displacement Analysis of the General Spatial 7-link 7R Mechanism. Mech. Mach. Theory 1988, 23, 219-226. 
(60) Manocha, D.; Canny, J. F. Efficient Inverse Kinematics for General 6R Manipulators. IEEE Trans. Rob. Autom. 1994 1994, 10, 648-657.

(61) Cortés, J.; Siméon, T.; Remaud-Siméon, M.; Tran, V. Geometric Algorithms for the Conformational Analysis of Long Protein Loops. J. Comput. Chem. 2004, 25, 956-967.

(62) Cortés, J.; Carrión, S.; Curcó, D.; Renaud, M.; Alemán, C. Relaxation of Amorphous Multichain Polymer Systems using Inverse Kinematics. Polymer 2010, 51, 4008-4014.

(63) Denarie, L.; Al-Bluwi, I.; Vaisset, M.; Siméon, T.; Cortés, J. Segmenting Proteins into Tripeptides to Enhance Conformational Sampling with Monte Carlo Methods. Molecules 2018, 23, 373-373.

(64) Dinner, A. R. Local Deformations of Polymers with Nonplanar Rigid Main-chain Internal Coordinates. J. Comput. Chem. 2000, 21, 1132-1144.

(65) Canutescu, A. A.; Jr., R. L. D. Cyclic Coordinate Descent: A Robotics Algorithm for Protein Loop Closure. Protein Sci. 2003, 12, 963-972.

(66) Li, Z.; Scheraga, H. A. Monte Carlo-Minimization Approach to the Multiple-Minima Problem in Protein Folding. Proc. Natl. Acad. Sci. 1987, 84, 6611-6615.

(67) Devaurs, D.; Molloy, K.; Vaisset, M.; Shehu, A.; Siméon, T.; Cortés, J. Characterizing Energy Landscapes of Peptides using a Combination of Stochastic Algorithms. IEEE Trans. Nano Biosci. 2015, 14, 545-552.

(68) Abraham, M. J.; Murtola, T.; Schulz, R.; Páll, S.; Smith, J. C.; Hess, B.; Lindahl, E. GROMACS: High Performance Molecular Simulations through Multi-Level Parallelism from Laptops to Supercomputers. SoftwareX 2015, 1, 19-25.

(69) Onufriev, A.; Case, D. A.; Bashford, D. Effective Born Radii in the Generalized Born Approximation: the Importance of being Perfect. J. Comput. Chem. 2002, 23, 12971304. 
(70) Becker, O. M.; MacKerell Jr, A. D.; Roux, B.; Watanabe, M. Computational Biochemistry and Biophysics; CRC Press: New York, 2001.

(71) Pettersen, E. F.; Goddard, T. D.; Huang, C. C.; Couch, G. S.; Greenblatt, D. M.; Meng, E. C.; Ferrin, T. E. UCSF Chimeraa Visualization System for Exploratory Research and Analysis. J. Comput. Chem. 2004, 25, 1605-1612.

(72) Vanquelef, E.; Simon, S.; Marquant, G.; Garcia, E.; Klimerak, G.; Delepine, J. C.; Cieplak, P.; Dupradeau, F.-Y. RED Server: a Web Service for Deriving RESP and ESP Charges and Building Force Field Libraries for New Molecules and Molecular Fragments. Nucleic Acids Res. 2011, 39, W511-W517.

(73) da Silva, A. W. S.; Vranken, W. F. ACPYPE-Antechamber Python Parser Interface. BMC Res. Notes 2012, 5, 367-367.

(74) Hunter, J. D. Matplotlib: A 2D Graphics Environment. Comput. Sci. Eng. 2007, 9, 90-95.

(75) Hayward, S. Peptide-Plane Flipping in Proteins. Protein Sci. 2001, 10, 2219-2227.

(76) Kufareva, I.; Abagyan, R. Homology Modeling; Springer, 2011; pp 231-257.

(77) Marelli, U. K.; Frank, A. O.; Wahl, B.; La Pietra, V.; Novellino, E.; Marinelli, L.; Herdtweck, E.; Groll, M.; Kessler, H. Receptor-Bound Conformation of Cilengitide Better Represented by Its Solution-State Structure than the Solid-State Structure. Chemistry-A European Journal 2014, 20, 14201-14206.

(78) Hruby, V. J.; Li, G.; Haskell-Luevano, C.; Shenderovich, M. Design of Peptides, Proteins, and Peptidomimetics in Chi Space. Pept. Sci. 1997, 43, 219-266.

(79) Geng, H.; Jiang, F.; Wu, Y.-D. Accurate Structure Prediction and Conformational Analysis of Cyclic Peptides with Residue-Specific Force Fields. J. Phys. Chem. Lett. 2016, 7, 1805-1810. 
(80) Slough, D. P.; Yu, H.; McHugh, S. M.; Lin, Y.-S. Toward Accurately Modeling NMethylated Cyclic Peptides. Phys. Chem. Chem. Phys. 2017, 19, 5377-5388. 